\title{
A Influência das Variáveis Meteorológicas na Ocorrência de Casos de Dengue em Fortaleza, Ceará
}

\author{
Patrícia Vieira Soares $^{1}$ (D), Roberto Alan Ferreira de Araújo ${ }^{1}$, Moisés Eudócio Almeida ${ }^{1}$ \\ Mestrado em Climatologia e Aplicações nos Países da CPLP, Universidade Estadual do Ceará, \\ Fortaleza, CE, Brasil.
}

Recebido em: 30 de Maio de 2021 - Aceito em: 5 de Novembro de 2021

\begin{abstract}
Resumo
Este trabalho tem por finalidade analisar a variabilidade climática e a sua relação com a dengue. O trabalho procurou correlacionar os dados meteorológicos coletados no site Instituto Nacional de Meteorologia - INMET relativo ao período de janeiro de 2014 a dezembro de 2019 e os dados do Sistema de Monitoramento Diário de Agravos (SIMDA) da Secretaria de Saúde de Fortaleza. Como resultado verificou-se que as variáveis meteorológicas que mais influenciam os casos de dengue na capital cearense são: velocidade do vento, temperatura mínima, temperatura máxima e precipitação e que o município de Fortaleza/Ce demonstra condições propícias para proliferação do mosquito Aedes aegypti no período em estudo.
\end{abstract}

Palavras-chave: variações climáticas, componentes principais, arbovirose, morbidades.

\section{The Influence of Meteorological Variables on the Occurrence of Dengue Cases in Fortaleza, Ceará}

\begin{abstract}
This work aims to analyze climate variability and its relationship with dengue. The work sought to correlate the meteorological data collected on the National Institute of Meteorology - INMET for the period from January 2014 to December 2019 and the data from the Daily Health Monitoring System (SIMDA) of the Health Department of Fortaleza. As a result, it was found that the meteorological variables that most influence dengue cases in the capital of Ceará are: wind speed, minimum temperature, maximum temperature and precipitation and that the municipality of Fortaleza/Ce demonstrates favorable conditions for the proliferation of the Aedes aegypti mosquito in the period under study.
\end{abstract}

Keywords: climatic variations, main components, arbovirus, morbidities.

\section{Introdução}

A interferência humana tem favorecido a extinção de muitas espécies, a degradação do meio ambiente e modificações no clima. Nos últimos anos, através do intenso processo de urbanização, áreas foram desmatadas e situações de acúmulo de água foram criadas, propiciando a disseminação de vetores como os mosquitos, que se adaptam a viver próximo aos humanos (Almeida et al., 2020).

Os diversos eventos associados ao clima podem influenciar a saúde humana, implicando no aumento de morbidades (Silva et al., 2014). A variabilidade climática desempenha uma grande influência na transmissão de doenças, especialmente nas transmitidas por vetores, como a leishmaniose, a malária e a dengue, que, somadas, correspondem a uma das causas consideráveis de mortes no Brasil e no mundo (Uchoa et al., 2019).

A dengue é uma doença infecciosa transmitida pelo Aedes aegypti, cuja dispersão pelos trópicos representa uma ameaça a saúde global, e, há poucos anos foi reconhecida pela Organização Mundial da Saúde como a patologia tropical de mais rápida proliferação em todos os continentes (Do et al., 2014). Seu vetor transmite pelo menos quatro enfermidades, as quais, dependendo da

Autor de correspondência: Patrícia Vieira Soares, pattyv.s@hotmail.com 
região podem ser: dengue, zika, chikungunya ou até mesmo febre amarela urbana (Galati et al., 2015).

Nos dias atuais, as notificações de dengue, tem sofrido um aumento crescente no Brasil destacando-se cinco anos epidêmicos 2008, 2010, 2013, 2015 e 2016, marcados pela reintrodução de novos sorotipos em $2010 \mathrm{e}$ 2013, bem como a introdução das novas arboviroses chikungunya e zika, notadas nos surtos de 2015 e 2016 (Ministério da Saúde, 2019).

As epidemias de dengue têm maior incidência nos meses mais quentes do ano, período do ápice reprodutivo do Aedes aegypti. A taxa de metabolismo do mosquito aumenta nos meses quentes, reduzindo seu ciclo em até oito dias, ou estendendo-se até 22 dias nos meses frios (Matiola e Ribeiro, 2019).

A falta de saneamento básico e de coleta dos resíduos são elementos importantes no aumento de criadouros disponíveis ao mosquito Aedes aegypt (Santos e Calado, 2014). Além disso, a urbanização desenfreada e sem planejamento, com consequente ampliação da densidade populacional, a baixa habilidade de mobilização social e dificuldade de acesso aos domicílios pelos agentes de saúde possibilitam condições favoráveis à transmissão do vírus (Fantinati et al., 2013).

No Brasil, a doença tem se apresentado como um dos principais problemas de saúde pública. Apesar de grandes investimentos e esforços por parte de autoridades governamentais de saúde, observa-se uma tendência crescente da incidência da doença com epidemias de grande relevância (Nascimento, 2016).

A introdução do vírus da dengue no município de Fortaleza/Ce se deu a partir de 1986 (Alves et al., 2017). Por ser uma das principais rotas turísticas do país, a cidade mantém as portas abertas à entrada de doenças e à circulação passiva de agentes transmissores (Lima et al., 2013). Este trabalho busca encontrar evidências que relacionem a variabilidade climática com os casos de dengue, na cidade de Fortaleza, durante o período de 2014 a 2019.

\section{Caracterização da Dengue}

O mosquito costuma atuar no início da manhã ou no final da tarde, tem voo baixo, ciclo de vida rápido e somente a fêmea transmite a doença pois ela é hematófaga, antropofílica e propaga o vírus da dengue quando pica um ser humano susceptível (Pego e Santos, 2014). Ao voar, o mosquito consegue chegar em calhas, terraços e caixas d'agua. A maioria das fêmeas Aedes aegypti pode passar a vida ao redor das casas onde emergem como adultos.

Esse mosquito prospera em centros urbanos e suburbanos devido as condições favoráveis nas residências e nos quintais (pneus, baldes, latas de água etc.) oferecendo condições ideais de reprodução para eles. Esse culicídeo desenvolveu um comportamento restritamente sinantrópico e antropogênico, sendo considerado a espécie de mosquito mais sujeita ao ambiente urbano (Almeida et al., 2020).

A dengue representa um dos principais problemas de saúde pública no mundo e afeta principalmente os países de clima tropicais, em virtude do clima quente e úmido, que forma condições ideais para a propagação do mosquito (Viana e Ignotti, 2013). É uma virose transmitida principalmente pela picada do mosquito do gênero Aedes, tendo como agentes causadores cinco variedades de vírus do grupo dos flavivírus: DENV-1, DENV- 2, DENV-3, DENV- 4 e DENV-5, que ainda não circula no Brasil (Simões, 2016). Um novo tipo de dengue (DENV-5) foi encontrado em 2007 por cientistas que analisavam amostras virais de dengue, o vírus foi colhido durante uma epidemia no estado de Sarawak, na Malásia, e percebeu-se que este é filogeneticamente distinto dos outros quatro tipos de dengue (Normile, 2013).

A dengue pode ser identificada pela ocorrência súbita de febre alta, acompanhada de fortes dores de cabeça, musculares e articulares, náusea, vômito e erupções cutâneas, podendo aparecer em 3 a 4 dias depois da febre (Pego e Santos, 2014). Apresenta também outros sintomas como: dores ósseas, coceira basicamente nas palmas e plantas, prostração, dor abdominal, diarreia, tonturas ao sentar ou levantar, que podem apontar queda de pressão arterial (hipotensão postural) e hemorragias induzidas ou espontâneas (Ministério da Saúde, 2016). A forma de manifestação viral se dará com a dengue com um dos quatro sorotipos. Quando o indivíduo tem contato com um desses vírus, o organismo ativa seu sistema de defesa e fica imune para uma das quatro manifestações dessa arbovirose (Furtado et al., 2019).

Calcula-se que 4 bilhões de pessoas estejam vivendo em regiões com risco de infecção pela doença e anualmente, 390 milhões de casos são registrados em todo mundo, dos quais 96 milhões se manifestam clinicamente, infelizmente, a dengue, afeta 128 países e é considerada uma doença negligenciada pela Organização Mundial da Saúde (MSF, 2020)

Segundo o Ministério da Saúde, a partir de 2014 ocorreu uma nova classificação de dengue passando agora a ter as seguintes denominações: dengue, dengue com sinais de alarme, e dengue grave (Costa e Calado, 2016). Quando o paciente apresenta um comprometimento orgânico grave: Como uma insuficiência hepática, cardíaca, alteração grave no sistema nervoso ou apresenta choque com uma queda importante de pressão arterial, será classificado como dengue grave (Ministério da Saúde, 2016).

\subsection{Dengue e variabilidade climática}

O quarto relatório de avaliação do painel intergovernamental sobre mudanças climáticas mostrou, em suas principais conclusões, os aspectos de impactos das mudanças climáticas na saúde humana, destacando-se: a 
manifestação de doenças e mortes prematuras; países pobres e as populações de baixa renda mais afetados; alterações nas temperaturas que causarão impactos diferenciados de acordo com as características regionais; variação no comportamento de vetores de doenças transmissíveis (Ministério da Saúde, 2009).

Estudos mostram que a temperatura do ar interfere na transmissão da dengue, pois atinge vários aspectos da vida do mosquito, tais como repasto sanguíneo das fêmeas, em sua longevidade e no período de incubação extrínseco do vírus (Donalísio et al, 2002; Beserra et al., 2014). É fato que níveis elevados de precipitação, temperatura do ar elevada, proximidades de periferias urbanas e baixa renda da população são fatores que favorecem a disseminação da doença (Sobral e Sobral, 2019).

As doenças epidêmicas mais afetadas pelos eventos climáticos são: malária, dengue, cólera e outras infecções de veiculação hídrica (WWF, 2015). Já no Brasil os casos de maior relevância associados aos extremos climáticos são: malária e dengue. Embora existam "evidências da influência do clima na ocorrência de algumas doenças, ainda não se conhecem todas as doenças sensíveis ao clima" (Sousa et al., 2018, p. 2).

O reaparecimento da dengue no Brasil se desenvolveu por uma inerente rede de variáveis ambientais, sociais e biológicas que, a partir de sua complexidade e dinamismo, estabeleceu o contexto prioritário para a ocorrência de sucessivas epidemias, resultando em um aumento do custo social e econômico para o país (Barbosa et al., 2017). As medidas de controle atuais têm por meta eliminar esse mosquito em suas diferentes fases; contudo, de modo geral, a efetividade dessas intervenções tem sido muito reduzida, não conseguindo conter a disseminação do vírus, de modo que as epidemias se sucedem, em grandes e, mais recentemente, também em pequenas cidades (Zara et al., 2016).

$\mathrm{O}$ rápido desenvolvimento e o não planejamento das cidades, condições de vida precárias, insuficiência da vigilância e do controle do vetor são alguns dos fatores relacionados à disseminação do vírus (Ferreira et al., 2018). Atualmente a doença manifesta extenso potencial epidêmico, avançando em todas as regiões do país, com notoriedade no Nordeste (Oliveira et al., 2018).

O estado do Ceará registrou aumentos de epidemias desde a da década de 1990 (Oliveira et al., 2018). Diante das condições ambientais favoráveis, do grande fluxo de turistas, da elevada densidade populacional, do suprimento irregular de água potável e baixas coberturas de saneamento básico, apresenta macrodeterminantes que corroboram para disseminação do vetor e prolongamento dos casos de dengue, com epidemias recorrentes (Cavalcanti et al., 2017).

A transmissão de várias doenças está relacionada com a ineficiência de políticas sanitárias. A ausência de sistemas de esgoto em muitas moradias contribui para a contaminação de águas subterrâneas e do solo (Silva et al., 2014). A atividade humana gera um acúmulo de resíduos, os quais trazem uma série de prejuízos ao meio ambiente $\mathrm{e}$ a saúde pública principalmente nas populações que não tem acesso ao saneamento básico.

Variáveis meteorológicas como: temperatura e umidade relativa do ar influenciam a procriação e a continuidade de agentes nocivos à saúde no meio ambiente, principalmente de mosquitos envolvidos na propagação de doenças como a dengue (Sousa et al., 2018). Vale ressaltar que mesmo um pequeno aumento na temperatura induzirá muitas espécies a se deslocarem dos seus habitats para latitudes e altitudes mais altas, modificando, assim, o padrão de incidência de várias doenças antes limitadas a determinadas regiões (Uchoa et al., 2019). A cidade de Fortaleza/Ce concentra o maior número de casos da doença do Estado do Ceará, por apresentar conjunturas ambientais caracterizadas por clima com elevadas temperaturas do ar e período invernoso concentrado na quadra chuvosa.

\section{Metodologia}

Neste estudo utilizou-se as informações obtidas no Sistema de Monitoramento Diário de Agravos (SIMDA), da Prefeitura de Fortaleza, a fim de obter dados de dengue no período de 2014 a 2019, as informações foram coletadas por mês, relacionando-as com os dados meteorológicos: temperatura (máxima, mínima) e umidade relativa do ar, amplitude térmica, velocidade do vento e precipitação. Obtidos na estação meteorológica do Instituto Nacional de Meteorologia (INMET) instalada em Fortaleza/Ce.

Para as análises das variáveis meteorológicas, executou-se a normalização dos dados para utilizar as técnicas estatísticas, considerando o método de Análise de Componentes Principais. Verificou-se a adequabilidade da amostra de variáveis através de testes em análise fatorial.

\subsection{Testes em análise fatorial}

Para verificar as variáveis meteorológicas que mais influenciam a dengue na cidade de Fortaleza, foram usados os seguintes testes:

1. Kaiser- Meyer- Olkin (KMO), é um critério para identificar se o modelo se ajusta aos dados. Os valores alcançados por meio deste método variam entre 0 e 1 , no qual 0,80 ou acima é admirável; 0,70 ou acima, mediano; 0,60 ou acima, medíocre; 0,50 ou acima, ruim; e abaixo de 0,50, inaceitável (Bakke et al., 2008).

2. Esfericidade de Bartlett's avalia em que medida a matriz de covariância é semelhante a uma matriz-identidade, onde os elementos da diagonal principal têm valor igual a um, e os demais elementos da matriz são próximos de zero, ou seja, não apresentam correlações 
entre si (Damásio, 2012). Os valores do teste de esfericidade de Bartlett's com níveis de significância $\mathrm{p}<0,05$ indicam que o teste é significativo (Dini et al., 2014).

3. As comunalidades representam a proporção da variância de explicação de cada indicador pelos componentes identificados. Para que o indicador seja representativo, seu valor de comunalidade deve ser maior que 0,6 (Dini et al., 2014). O valor próximo de 1 irá explicar todas as outras variáveis.

\subsection{Análise de componentes principais}

A técnica estatística de Análise de Componentes principais (ACP) tem como uma de suas metas diminuir a dimensionalidade do sistema, por meio da definição de uma nova base, cujos componentes são as Componentes Principais, obtidas a partir da matriz de covariâncias das variáveis originais (Rossi, 2017). Essa redução da dimensionalidade dos dados acontece sem que ocorra grande perda das informações, mantendo ao máximo a variação presente nos dados (Graniti, 2012).

Os componentes principais denotam propriedades importantes: cada componente principal é uma combinação linear de todas as variáveis originais, são independentes entre si e determinados com o propósito de reter, em ordem de estimação, a maior quantidade de informação, em termos da variação total contida nos dados (Varella, 2008). Geralmente, elege-se o componente principal de maior importância (o primeiro componente principal), como sendo aquele de maior variância, que explique o máximo de variabilidade dos dados; o segundo componente de maior relevância, o que apresenta a segunda maior variância, e assim continuamente, até o componente principal de menor importância (Sampaio et al., 2017).

A análise de componentes principais é usada para extrair informações importantes de uma tabela de dados multivariada e expressar as informações como um conjunto de novas variáveis chamadas de componentes prin- cipais. Nessa ferramenta, o pesquisador poderá selecionar as variáveis mais pertinentes no cálculo da ACP (Rossi, 2017). Essas novas variáveis correspondem a combinações lineares das variáveis originais.

$\mathrm{Na}$ análise de componentes principais, as variáveis costumam ser escalonadas ou seja, padronizadas. Isso é especialmente recomendado quando as variáveis são medidas em escalas diferentes; caso contrário, as saídas ACP obtidas serão severamente afetadas (Oper Data, 2021). Vale ressaltar que a "abundância de dados pode dificultar a compreensão de como as diferentes variáveis analisadas se relacionam" (Rossi, 2017, p. 1).

Obteve-se resultados que farão parte da discussão em torno dos parâmetros da morbidade dependente (dengue), e as variáveis meteorológicas que assumem o papel de variáveis independentes.

\section{Resultados e Discussões}

Foram coletados dados da morbidade dengue e acumulados de precipitação (Fig. 1), que representam uma amostra geral da cidade. A série validada por esses dados foi feita através dos totais observados durante o período de janeiro de 2014 a dezembro de 2019.

No período de janeiro de 2014 a dezembro de 2019 foram confirmados 71.529 casos de dengue. Os meses com maior índice da doença foram: junho de 2014 com 1.097 casos, maio de 2015 com 10.236 casos, maio de 2016 com 5.846 casos, abril de 2017 com 4.531 casos, abril 2018 com 370 casos e abril de 2019 com 617 casos. $\mathrm{O}$ ano que apresentou o maior número de casos confirmados foi 2015 com 26.437 casos. Vale ressaltar que o aumento de casos dengue coincidiu com a quadra chuvosa no período de fevereiro a maio.

Suas variações temporais são mostradas, temperatura do ar (máxima e mínima) (Fig. 2) e velocidade do vento no (Fig. 3).

Nas séries temporais os dados são observados em diferentes instantes de tempo, essa investigação foi reali-

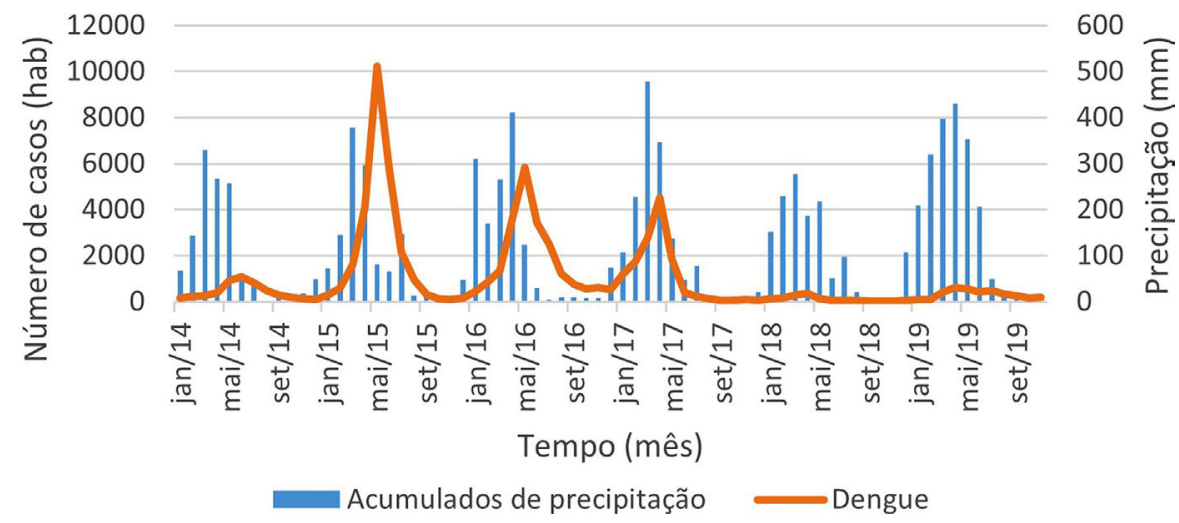

Figura 1 - Casos de dengue e acumulados de precipitação entre os anos de 2014 a 2019 em Fortaleza/Ce. Fonte: Sistema de Monitoramento Diário de Agravos e INMET - Instituto Nacional de Meteorologia. 


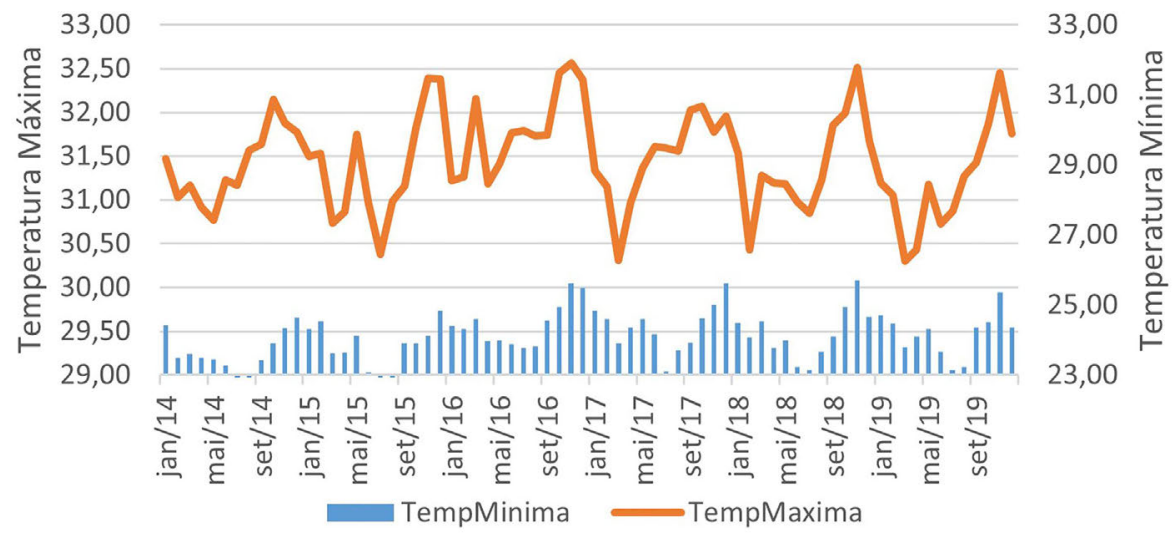

Figura 2 - Temperatura do ar (máxima e mínima) em Fortaleza/Ce, no período de 2014 a 2019. Fonte: INMET - Instituto Nacional de Meteorologia.

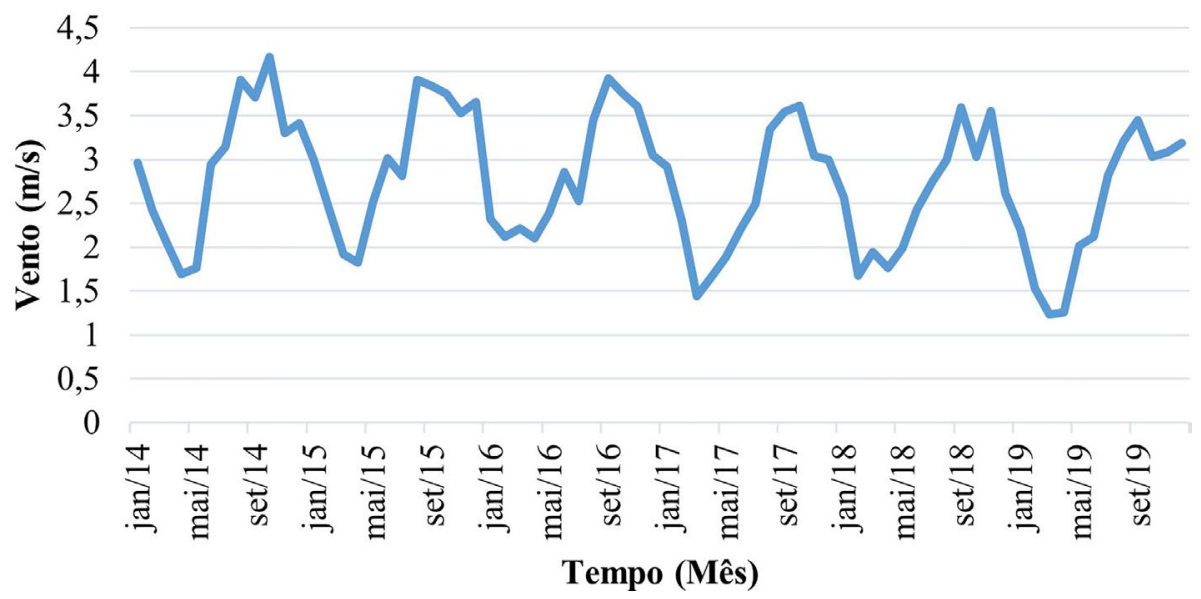

Figura 3 - Velocidade do Vento entre os anos de 2014 a 2019 em Fortaleza/CE. Fonte: INMET - Instituto Nacional de Meteorologia.

zada através da normalização dos dados, ou seja, visando definir se estas grandezas estão abaixo ou acima da média.

Vale ressaltar que a temperatura mínima durante o período do estudo variou de $22,34^{\circ} \mathrm{C}$ a $25,70^{\circ} \mathrm{C}$, já a temperatura máxima variou de $30,30^{\circ} \mathrm{C}$ a $32,56^{\circ} \mathrm{C}$.

Para verificar se a modelagem utilizada seria satisfatória utilizou-se os testes KMO, esfericidade Bartlett's e a comunalidade, no KMO, foi alcançado o valor entre $0.6 \mathrm{e}$ 0.8 , que dentro da análise mostra que, mesmo sendo um valor médio, podemos utilizar pois está dentro dos parâmetros de aceitabilidade, já o teste de esfericidade Bartlett's trouxe valores próximos a 0 , confirmando a eficiência do método, como mostra a Tabela 1.

$\mathrm{Na}$ comunalidade pode-se observar que nem todas as variáveis consegue explicar a dengue, a umidade relativa e

Tabela 1 - Teste KMO e Bartlett's.

\begin{tabular}{lc}
\hline Kaiser-Meyer-Olkin measure of sampling adequacy & 0,6 \\
Bartlett's test of sphericity approx. chi-square & 265,6 \\
Df & 21 \\
Sig. & 0,0 \\
\hline
\end{tabular}

amplitude, são as que menos explicam a doença como mostra a Tabela 2. De uma maneira direta, quanto maior o valor de comunalidade, maior o poder de explicação daquela variável em função das outras em questão na observação.

O total da variância explicada foi um acumulativo de $66,63 \%$ observado na Tabela 3 pelo método de ACP o que gerou o teste de inercia, sendo possível observar duas componentes explicitamente, velocidade do vento e temperatura mínima, que explicam a observação da rodada

Tabela 2 - Comunalidades.

\begin{tabular}{lcc}
\hline & Inicial & Extração \\
\hline Dengue & 1,0 & 0,1 \\
Temperatura mínima & 1,0 & 0,8 \\
Temperatura máxima & 1,0 & 0,8 \\
Amplitude & 1,0 & 0,6 \\
Velocidade do vento & 1,0 & 0,8 \\
Precipitação & 1,0 & 0,8 \\
Umidade relativa & 1,0 & 0,5 \\
\hline
\end{tabular}


Tabela 3 - Total da variância explicada.

\begin{tabular}{|c|c|c|c|c|c|c|c|c|c|}
\hline \multirow[t]{2}{*}{ Componentes } & \multicolumn{3}{|c|}{ Autovalores iniciais } & \multicolumn{3}{|c|}{ Extração de somas de cargas quadradas } & \multicolumn{3}{|c|}{ Soma de rotação de cargas quadradas } \\
\hline & Total & \% de Variância & Cumulativa \% & Total & \% de Variância & Cumulativa \% & Total & \% de Variância & Cumulativa \% \\
\hline 1 & 3,10 & 44,40 & 44,40 & 3,10 & 44,40 & 44,40 & 2,95 & 42,21 & 42,21 \\
\hline 2 & 1,55 & 22,23 & 66,63 & 1,55 & 22,23 & 66,63 & 1,71 & 24,42 & 66,63 \\
\hline 3 & 0,95 & 13,62 & 80,26 & & & & & & \\
\hline 4 & 0,61 & 8,74 & 89,01 & & & & & & \\
\hline 5 & 0,53 & 7,67 & 96,69 & & & & & & \\
\hline 6 & 0,13 & 1,89 & 98,59 & & & & & & \\
\hline 7 & 0,09 & 1,40 & 100,00 & & & & & & \\
\hline
\end{tabular}

efetivada. Com isso pode-se afirmar que é real, devido aos altos valores que foram truncados maiores que 1 , sendo a primeira componente com uma variância acima de $44 \%$ e a segunda componente acima de $22 \%$ como segue a Tabela 3 .

Assim, foi obtida a matriz das componente não rotacionadas e rotacionadas no espaço euclidiano, onde verificamos que o melhor ajuste se dá com a rotação das mesmas, visto espacialmente na Fig. 4, que faz uma divisão espacial em níveis das componentes, mostrando uma relação da variável dependente proporcional com a precipitação (vindo a confirmar a dependência dessa para sua variância), e inversamente proporcional com as variáveis de temperatura, sendo que a UR se mostrou atuante também neste contexto de dependência como verificado na
Fig. 4. Isso nos mostra o valor dessas variáveis têm diretamente explicação de dengue no município de Fortaleza.

Visto isso, os resultados mostram que a modelagem abordada tem condições de ser utilizada para esta finalidade, salientando que mesmo em alguns momentos os resultados são de baixa consistência.

\section{Considerações Finais}

Nesse estudo foi constatado que houve um aumento significativo de casos de dengue em Fortaleza nos últimos anos. A cidade experimenta epidemias de dengue desde 1986, as quais estão associadas a variabilidade climática, o que justifica a necessidade de estudar a influência das va-

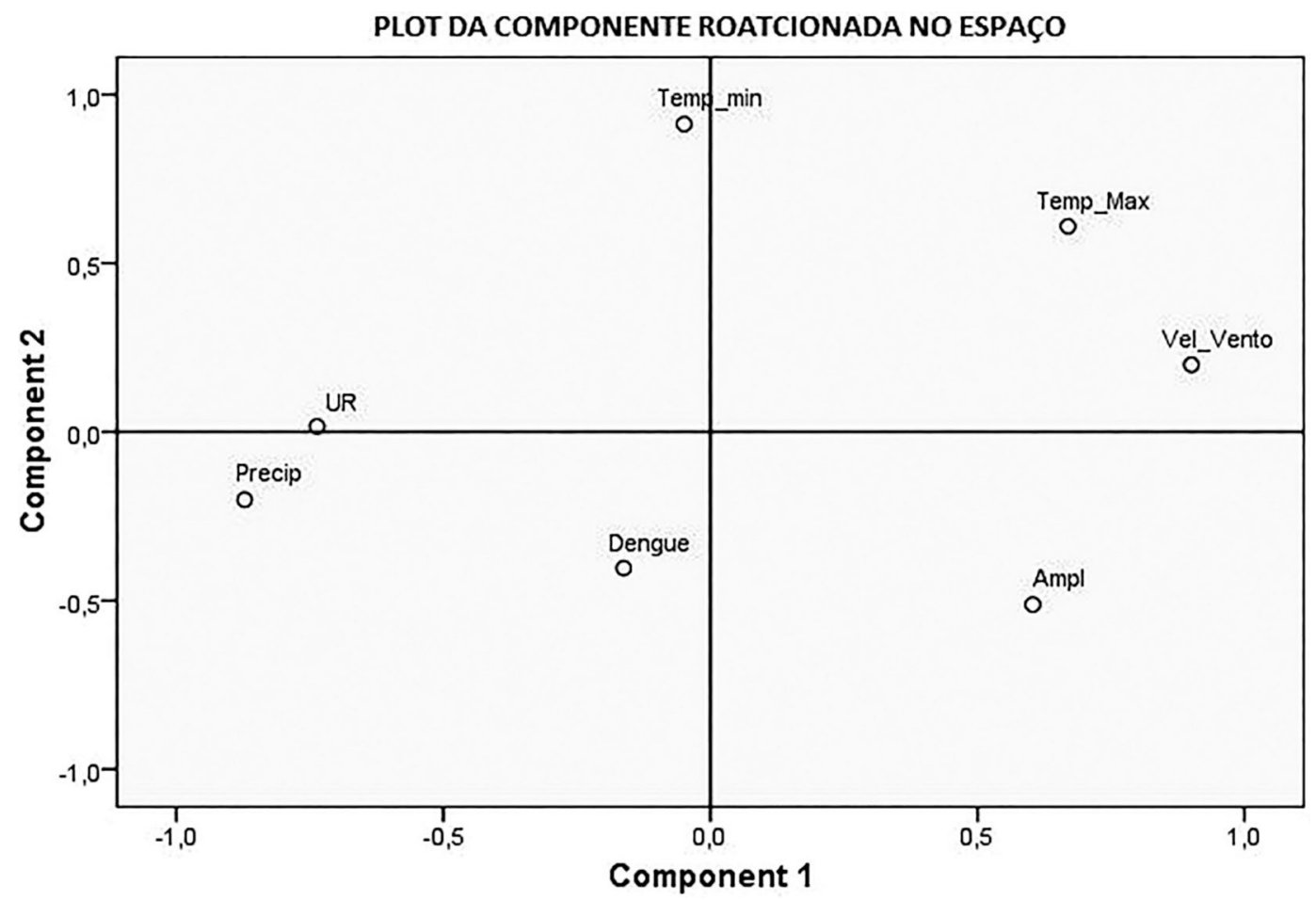

Figura 4 - Plot da componente em espaço rotacionado. 
riáveis meteorológicas na ocorrência de casos de dengue em Fortaleza, Ceará.

Conseguiu verificar que a estatística multivariada, pode ser aplicada como meio de acompanhamento das variáveis meteorológicas que mais influenciam os casos de dengue na capital cearense e de acordo com as variáveis estudadas, o Município demonstra condições propícias para proliferação do mosquito Aedes aegypti, assim como transmissão da dengue.

Verificou-se a influência das variáveis meteorológicas utilizando os testes de Kaiser-Mayer-Olklin (KMO) e esfericidade de Bartlett's e comunalidade. O teste KMO mostrou-se está dentro da aceitabilidade, sendo ratificado pelo teste de esfericidade de Bartlett's que apresentou pvalor inferior a 0,05 , o que indicam que as correlações entre as variáveis são suficientes para a realização do estudo. Comprovou-se pela comunalidade, que as variáveis meteorológicas que mais explicam a dengue no Município de Fortaleza são: temperatura mínima, temperatura máxima, velocidade do vento e precipitação e as que menos explicam a dengue são umidade relativa seguida da amplitude térmica.

Durante o trabalho verificou-se que várias doenças veiculadas por mosquitos estão associadas a eventos meteorológicos e que o aumento na temperatura pode aumentar a quantidade e reduzir o tempo de desenvolvimento desses indivíduos.

O auxílio de modelagens é de grande relevância para o melhor entendimento das particularidades que tem como foco o Aedes aegypti, porém, há uma grande complexidade das relações entre fatores climáticos e as arboviroses, necessitando de estudos mais avançados e complementares para entender esses fenômenos.

Os resultados apresentados indicam para um panorama preocupante, pois o conjunto das variáveis meteorológicas, questões sociais e a facilidade de adaptação do mosquito ao ambiente corroboram para a disseminação do Aedes aegypti, favorecendo assim a prevalência de casos de dengue.

\section{Referências}

ALMEIDA, L.S.; COTA, A.L.S.; RODRIGUES, D.F. Análise climática e a distribuição dos casos de dengue em Fortaleza. In: Anais XVII Simpósio Brasileiro de Geografia Aplicada. Campinais: UNICAMP, p. 2710-2714, 2020.

ALVES, R.J. Saneamento, arboviroses e determinantes ambientais: Impactos na saúde urbana. Revista Ciência e Saúde Coletiva, v. 25, n. 10, p. 2710-2714, 2021.

BAKKE, H.A.; LEITE, A.S.; SILVA, L.B. Estatística multivariada: aplicação da análise fatorial na engenharia de produção. Revista Gestão Industrial, v. 4,n, 4, p. 01-14, 2008.

BARBOSA, I.R.; TAVARES, A.M.; TORRES, U.P.S. NASCIMENTO, C.A. MOURA, M.C.B.M., et al. Identificação de áreas prioritárias para a vigilância e controle de dengue e outras arboviroses transmitidas pelo Aedes aegypti no município de Natal-RN: relato de experiência. Revista Epidemiologia e Serviços de Saúde, v. 26, n. 3, p. 629638, 2017.

BESERRA, E.B.; RIBEIRO, P.S.; OLIVEIRA, S.A. Flutuação populacional e comparação de métodos de coleta de Aedes (stegomyia) aegypti (diptera, culicidae). Revista Iheringia - Serie Zoologia, v. 104, n. 4, p. 418-425, 2014.

CAVALCANTI, L.P; BARRETO, F.K.A; CANUTO, I.F.P; LIMA, A.A; LIMA, J.W.O., et al. Trinta anos de dengue no Ceará: história, contribuições para ciência e desafios no cenário atual com tripla circulação de arbovírus. Journal of Health \& Biological Sciences, v. 6, n. 1, p. 65, 2017.

COSTA, I.M.P.; CALADO, D.C. Incidência dos casos de dengue (2007-2013) e distribuição sazonal de culicídeos (20122013) em Barreiras, Bahia. Periódico Epidemiologia e Serviços de Saúde, v. 25, n. 4, p. 735-744, 2016.

DAMÁSIO, B.F. Uso da análise fatorial exploratória em psicologia. Periódico Avaliação Psicológica, v. 11, n. 2, p. 213228, 2012.

DINI, A.P; ALVES, D.F; OLIVEIRA, H.C; GUIRARDELLO, E.B. Validade e confiabilidade de um instrumento de classificação de pacientes pediátricos. Revista Latino-Americana de Enfermagem, v. 22, n. 4, p. 598-603, 2014.

DO, T.T.T; MARTENS, P.; LUU, N.H; WRIGHT, P; CHOISY, M., et al. Climatic-driven seasonality of emerging dengue fever in Hanoi, Vietnam. BMC Public Health, v. 14, n. 1, p. 1-10, 2014.

DONALÍSIO, M.R.; GLASSER, C.M. Vigilância entomológica e controle de vetores do dengue.Revista Brasileira de Epidemiologia, v. 5, n. 3, p. 259-279, 2002.

FANTINATI, A.M.M; SANTOS, A.C; INUMARU, S.S; DUARTE, V.T; FANTINATI, M.S., et al. Perfil epidemiológico e demográfico dos casos de dengue na região central de Goiânia - Goiás. Revista Tempus Actas Saúde Col, v. 1, n. 1, p. 107-119, 2013.

FERREIRA, A.; CHIARAVALLOTI, F.; MONDINI, A. Dengue em Araraquara, SP: epidemiologia, clima e infestação por Aedes aegypti. Revista de Saúde Pública, v. 52, n.18, p. 110, 2018.

FURTADO, A.N.R; LIMA, A.S; OLIVEIRA, A.S; TEIXEIRA, A.B; FERREIRA, D.S., et al. Dengue e seus avanços. Revista Brasileira de Análises Clínicas, v. 51, n. 3, p. 1-9, 2019.

GRANITI, R. Análise de Componentes Principais da iniciação da Marcha em Pacientes com Síndrome de Parkison. Dissertação de Mestrado em Engenharia Biomédica, Programa de Pós-Graduação em Engenharia Biomédica, Universidade Federal do Rio de Janeiro, Rio de Janeiro, 89 p., 2012.

INSTITUTO NACIONAL DE METEOROLOGIA DO BRASIL. Normais Climatológicas (2014/2019). Brasília: INMET, 2020.

MATIOLA, C.; RIBEIRO, E.A.W. Análise exploratória dos dados climáticos e sua influência no aedes aegypti, no município de Chapecó/SC. Revista Brasileira de Geografia Médica e da Saúde, v. 15, n. 33 p. 29-41, 2019.

MINISTÉRIO DA SAÚDE. Mudança Climática e Saúde: Um Perfil do Brasil. Brasília: Organização Pan-Americana da Saúde, 2009. 
MINISTÉRIO DA SAÚDE. Dengue: Diagnóstico e Manejo Clínico Adulto e Criança. Brasília: Secretaria de Vigilância em Saúde Secretaria de Atenção à Saúde, 2016.

MINISTÉRIO DA SAÚDE. Boletim Epidemiológico. Brasília: Secretaria de Vigiláncia em Saúde, v. 50, p. 1-154, 2019.

MSF. Dengue. Disponível em https://www.msf.org.br/o-quefazemos/atividadesmedicas/dengue?utm_source $=\mathrm{ad}$ words_msf\&utm_medium $=\& u t m \_$campaign $=$dengue_co municacao\&utm_content $=$ exclusao-saude_bra sil_39923\&gclid=EAIaIQobChMIi5vF8cmN6QIV1 wyRCh3bKQfXEAAYASAAEgIv-vD_BwE, acesso em 20 de jun. de 2020.

NASCIMENTO, L.B. Dengue em Gestantes e a Associação entre a Infecção Sintomática e Desfechos Desfavoráveis em Nascidos Vivos: Um Relacionamento entre os Dados dos Sistemas de Informação em Saúde no Brasil. Tese de Doutorado em Medicina Tropical e Saúde Pública, Programa de Pós-Graduação em Medicina Tropical e Saúde Pública, Universidade Federal de Goiás, Goiania, 261 p., 2016.

NORMILE, D. First New Dengue Virus Type in 50 Years. Disponível em https:/www.sciencemag.org/news/2013/10/ first-new-dengue-virus-type-50-years, acesso em 20 de jun. de 2020.

OLIVEIRA, R.M.; ARAÚJO, F.M.C.; CAVALCANTI, L.P.G. Aspectos entomológicos e epidemiológicos das epidemias de dengue em Fortaleza, Ceará, 2001-2012. Revista do Sistema Único de Saúde do Brasil, v. 27, n. 1, p. 1-10, 2018.

OPER DATA. Análise de Componentes Principais. Disponível em https://operdata.com.br/blog/analise-de-componentesprincipais/, acesso em 20 de jun. de 2021.

PEGO, C.; SANTOS, V; LIMA, V. A dengue. Revista FSP, v. 1, n. 1, p. 1-12, 2014.

ROSSI, R.G. Análise de Componentes Principais em Data Warehouses. Dissertação de Mestrado em Ciências da Computação, Programa de Pós-Graduação em Ciências da Computação, Universidade de São Paulo, São Paulo, 81 p., 2017.

SAMPAIO, S.A; HONGYU, K; PINTO, J.M; SOARES, V.E; NAVARRO, R.S., et al. Utilização da análise multivariada para dados de hepatites virais B E C: Parte 1. Revista de Enfermagem, v. 11, n. 8, p. 3021-3030, 2017.

SANTOS, I.M.; CALADO, D. Captura de mosquitos antropofílicos (Diptera, Culicidae) em uma área urbana da região oeste da Bahia, Brasil. Revista Iheringia, Série Zoologia, v. 104, n. 1, p. 32-38, 2014.

SANTOS, T.O; ANDRADE, V.S; ROCHA, V.M; MENEZES, J.S. Os impactos do desmatamento e queimadas de origem antrópica sobre o clima da Amazônia brasileira: um estudo de revisão. Revista Geográfica Acadêmica, v. 11, n. 2, p. 157-181, 2017.

SILVA, D.D; MIGLIORINI, R.B; SILVA, E.C; LIMA, Z.M; MOURA, I.B., et al. Falta de saneamento básico e as águas subterrâneas em aquífero freático: Região do bairro Pedra Noventa, Cuiabá (MT). Revista de Engenharia Sanitária e Ambiental, v. 19, n. 1, p. 43-52, 2014.

SILVA, E.N.; RIBEIRO, H.; SANTANA P. Clima e saúde em contextos urbanos: uma revisão da literatura. Revista Bibliográfica de Geografía Y Ciencias Sociales, v. 19, n. 1092, p. 1-24, 2014.

SIMDA - Secretaria Municipal de Saúde de Fortaleza. Sistema de Monitoramento Diário de Agravos. Disponível em https://simda.sms.fortaleza.ce.gov.br/simda/index, acesso em 6 jul. 2020.

SIMÕES, L.B. Avaliação da Gravidade da Dengue Segundo o Nível de Intervenção Clínica e Avaliação Ultrassonográfica dos Casos em Crianças e Adultos. Tese de Doutorado em Medicina Tropical, Programa de Pós-Graduação em Medicina Tropical, Universidade Federal de Pernambuco, Recife, 164 p., 2016.

SOBRAL, M.F.F.; SOBRAL, A.I. Casos de dengue e coleta de lixo urbano: Um estudo na cidade do Recife, Brasil. Revista de Ciência e Saúde Coletiva, v. 24, n. 3, p. 1075-1082, 2019.

SOUSA, T.C.M; AMANCIO, F; HACON, S.S; BARCELLOS, C. Doenças sensíveis ao clima no Brasil e no mundo: Revisão sistemática. Revista Panamericana de Salúde Publica, v. 42, n. 85, p. 1-10, 2018.

UCHOA, N.M.; UCHOA, M.; LUSTOSA, R.P. Relação entre mudanças climáticas e saúde humana. Revista Revinter, v. 12, n. 1, p. 11-18, 2019.

VARELLA, C.A. Análise multivariada aplicada as ciências agrárias análise. Revista de Agronomia, v. 1, n. 1, p. 1-12, 2008.

VIANA, D.V.; IGNOTTI, E. A ocorrência da dengue e variações meteorológicas no Brasil: Revisão sistemática. Revista Brasileira de Epidemiologia, v. 16, n. 2, p. 240-256, 2013.

WWF. As Mudanças Climáticas: Riscos e Oportunidades. Brasília: Editora Água Brasil, 2015.

ZARA, A.L; SANTOS, S.M; OLIVEIRA, E.S; CARVALHO, R.G; COELHO, G.E., et al. Estratégias de controle do Aedes aegypti: Uma revisão. Epidemiologia e serviços de saúde. Revista do Sistema Único de Saúde do Brasil, v. 25, n. 2, p. 391-404, 2016.

License information: This is an open-access article distributed under the terms of the Creative Commons Attribution License (type CC-BY), which permits unrestricted use, distribution and reproduction in any medium, provided the original article is properly cited. 